\title{
Region Filter and Optical Flow based Video Surveillance System
}

\author{
Dolley Shukla \\ Shri Shankaracharya College of Engg. \& Tech
}

\author{
Surabhi Biswas \\ Shri Shankaracharya College of Engg. \& Tech.
}

\begin{abstract}
During the last few years different video-surveillance systems have been developed based on video processing and using different techniques. This surveillance system generally seeks to track people (and/or vehicles) moving through a scene, to classify the behaviors of each track, and to identify whether these behaviors can be considered normal or abnormal. All Automated surveillance systems require some mechanism to detect interested objects in the field of view of the sensor. Once objects are detected, the further processing for tracking. In my paper a method is described for tracking moving objects from a sequence of video frame. This method is implemented by using optical flow (Horn-Schunck) and Region filtering in matlab simulink. The objective of this paper is to identify and track a moving object within a video sequence for both Abrupt change video as well as Gradual change video in video surveillance.
\end{abstract}

Keywords : Optical flow, Region filtering, Threshold, Simulink.

\section{INTRODUCTION}

During the last few years different video-surveillance systems have been developed based on video processing and using different techniques. The Video - surveillance systems, normally using imagery from CCTV cameras as their input

\begin{abstract}
can range from simple movement detection equipment to very sophisticated "intelligent" scene analysis devices.[1]

Video is the technology of electronically capturing, recording, processing, storing, transmitting, and reconstructing a sequence of still images representing scenes in motion. Surveillance is the monitoring of the behavior activities, or other changing information, usually of people for the purpose of influencing, managing, directing, or protecting.[1]
\end{abstract}

The use of video camera to transmit a signal to a specific place, on a limited set of monitors is called Video Surveillance. Interest in video surveillance techniques has grown significantly in the years. This interest has led to a surge of Closed Circuit Television (CCTV) cameras being installed in public areas for surveillance-a departure from the traditional use by private companies for property protection.[2]

This application generally seeks to track people (and/or vehicles) moving through a scene, to classify the behaviors of each track, and to identify whether these behaviors can be considered normal or abnormal (significant). So in this paper we are using optical flow analysis and Blob analysis to determine changes occur in scene of video [1].

\section{VIDEO SURVILLANCE}

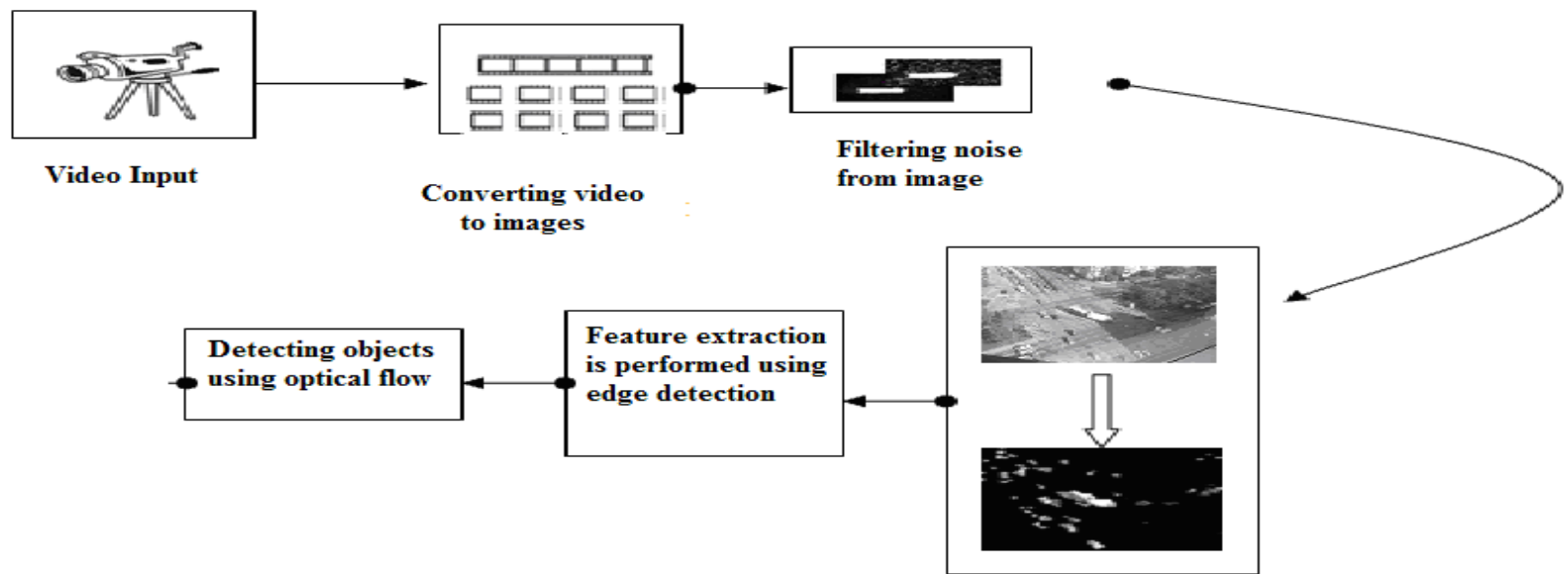

Segmentation based on

Background subtraction

Fig 1: Architecture of the implementation of video surveillance

The main objective of the Video Surveillance is to detect and track the moving object and changes in scene occur through video sequence. Here the framework is carried out using the image processing steps such as; Video processing, frame display, background subtraction, edge Detection, segmentation and finally the object is detected and then tracked. 


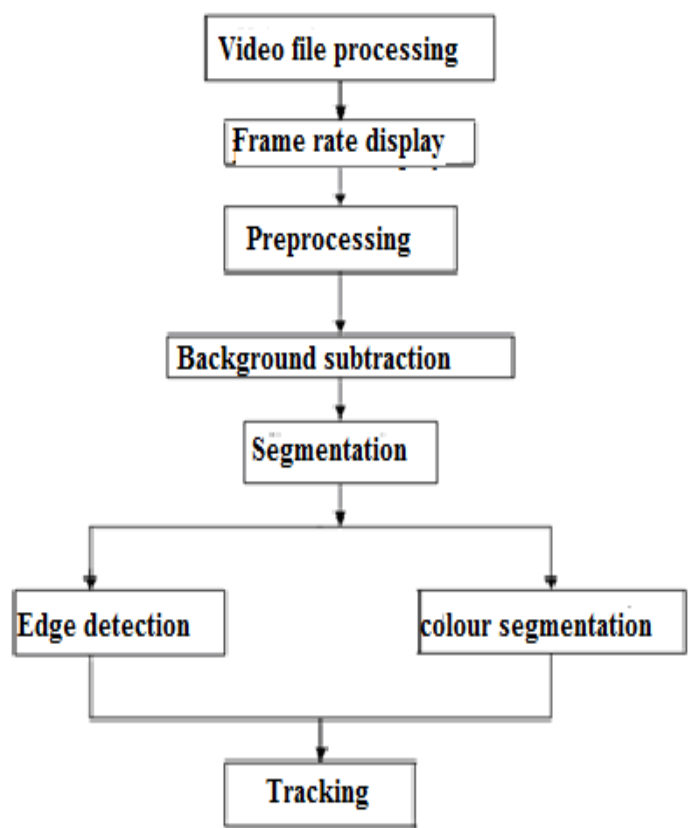

Fig 2: Flow Chart of Video Surveillance

First, the videos are separated as frames and pre-processing method is used for the colour conversion to subtract the foreground objects from the background, and background subtraction is used to find the total or sudden change in intensity in the video. Edge detection is performed as the middle wok and to extract the boundary of the object from the background segmentation module is executed. Finally tracking will be carried out. [3]

This proposed framework combines the existing and recent techniques of video processing techniques for Video Surveillance system. The output of the system delivers a simulink -based application development platform intended for Video Surveillance system.
Pre-processing - Pre-processing is mainly used to enhance the contrast of the image, removal of noise and isolating objects of interest in the image. Pre-processing is any form of signal processing for which the output is an image or video. [4]

Background subtraction - It is a computational vision process of extracting foreground objects in a particular scene. The automated surveillance systems require some mechanism to detect interested objects in the field of view of the sensor. Once objects are detected, the further processing for tracking and activity is limited in the corresponding regions of the image. In vision based systems following detection methods are used one of which is background subtraction this method takes initial frame as the base frame and subtracts all the other frames from it. [5]

Segmentation - The processing of subdividing the image into its constituent parts or object is called image segmentation. [6] Segmentation based on Edge Detection -

Edge detecting is a collection of very important local image pre-processing method used to locate changes in the intensity function. An edge is a property attached to an individual pixel and is calculated from the image function behaviors in a neighborhood of that pixel. Edge takes a gray scale image as its input, and returns a binary image of the same size as the input image, with 1's where the function finds edges and 0's elsewhere [6]. There are various different Operators of edge detection they are -

Roberts operators

Prewitt Operator

Sobel operator

$\square$ Canny operator

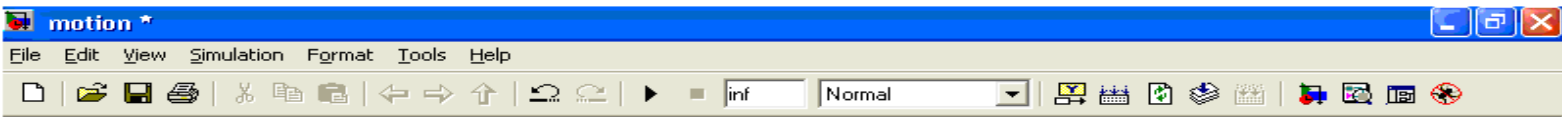

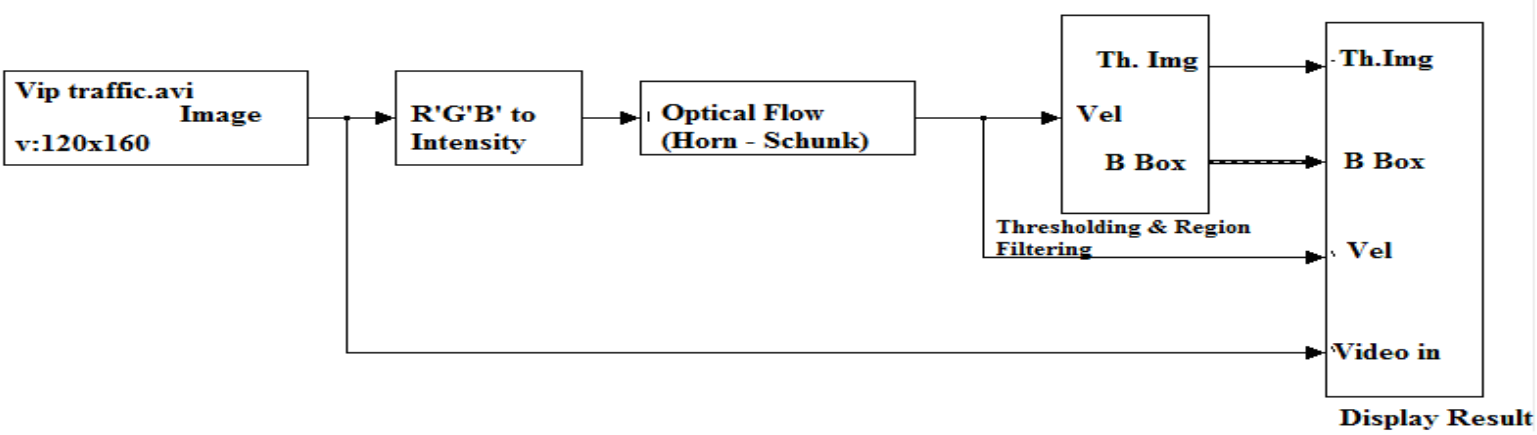

Fig 3 : Simulik based block diagram of video survillance

\section{RGB BLOCK}

RGB Block is Colour Space Conversion block converts colour information between colour spaces. Using the Conversion parameter to specify the colour spaces we are converting RGB as $\mathrm{R}^{\prime} \mathrm{G}^{\prime} \mathrm{B}^{\prime}$ to $\mathrm{Y}^{\prime} \mathrm{CbCr}, \mathrm{Y}^{\prime} \mathrm{CbCr}$ to $\mathrm{R}^{\prime} \mathrm{G}^{\prime} \mathrm{B}$,
R'G'B' to intensity, R'G'B' to HSV, HSV to R'G'B', sR'G'B' to $X Y Z, X Y Z$ to sR'G'B', sR'G'B' to sR'G'B' to $L^{*} a^{*} b^{*}$, and $L^{*} a^{*} b^{*}$ to $s R^{\prime} G^{\prime} B^{\prime}$ [7]. It provides a set of routines for converting between RGB and other colour spaces. The image processing functions themselves assumes all colour data in RGB and process an image that uses a different colour space 
by first converting it to RGB, and then converting the processed image back to the original colour space.

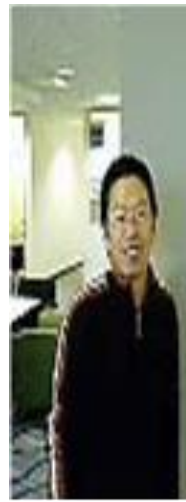

(a)
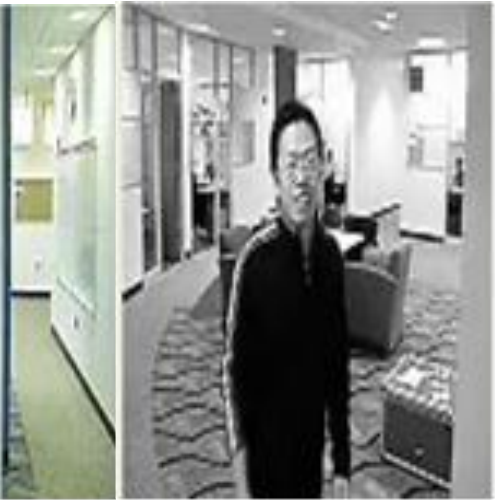

(b)
Fig 4: Result of RGB block: (a) Original Image (b) Intensity image

\section{OPTICAL FLOW}

Optical flow is a technique which determines the "distribution of apparent velocities of movement of brightness patterns in an image". The Optical Flow block estimates the direction of object motion from one image to another or from one video frame to another using either the Horn-Schunk or the the Lucas-Kanade method. In these we are using Horn-Schunk method as it effectively minimizes distortions in flow thus giving solutions which show more smoothness [9].

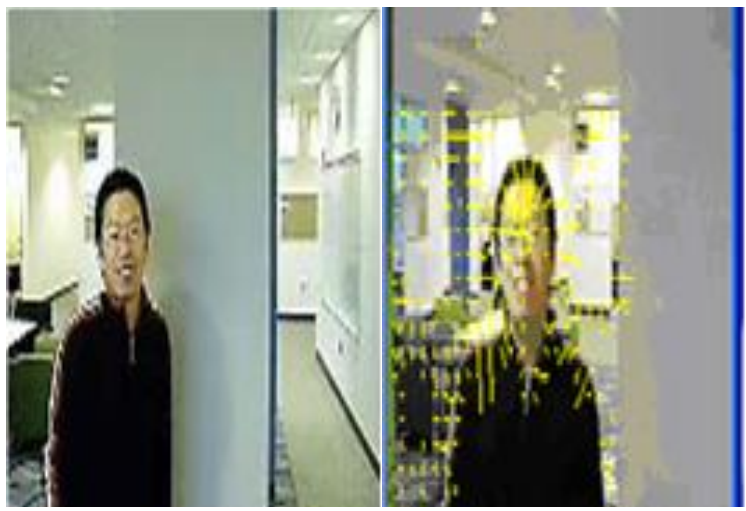

(a)

(b)

Figure 5 : O/P of Optical flow , (a) original video (b) Result of optical flow

\section{THRESHOLDING \& REGION FILTERING}

Thresholding is a fundamental method to convert a gray scale image into a binary mask, so that the objects of interest are separated from the background. Region filtering is used to extracts moving object from video.

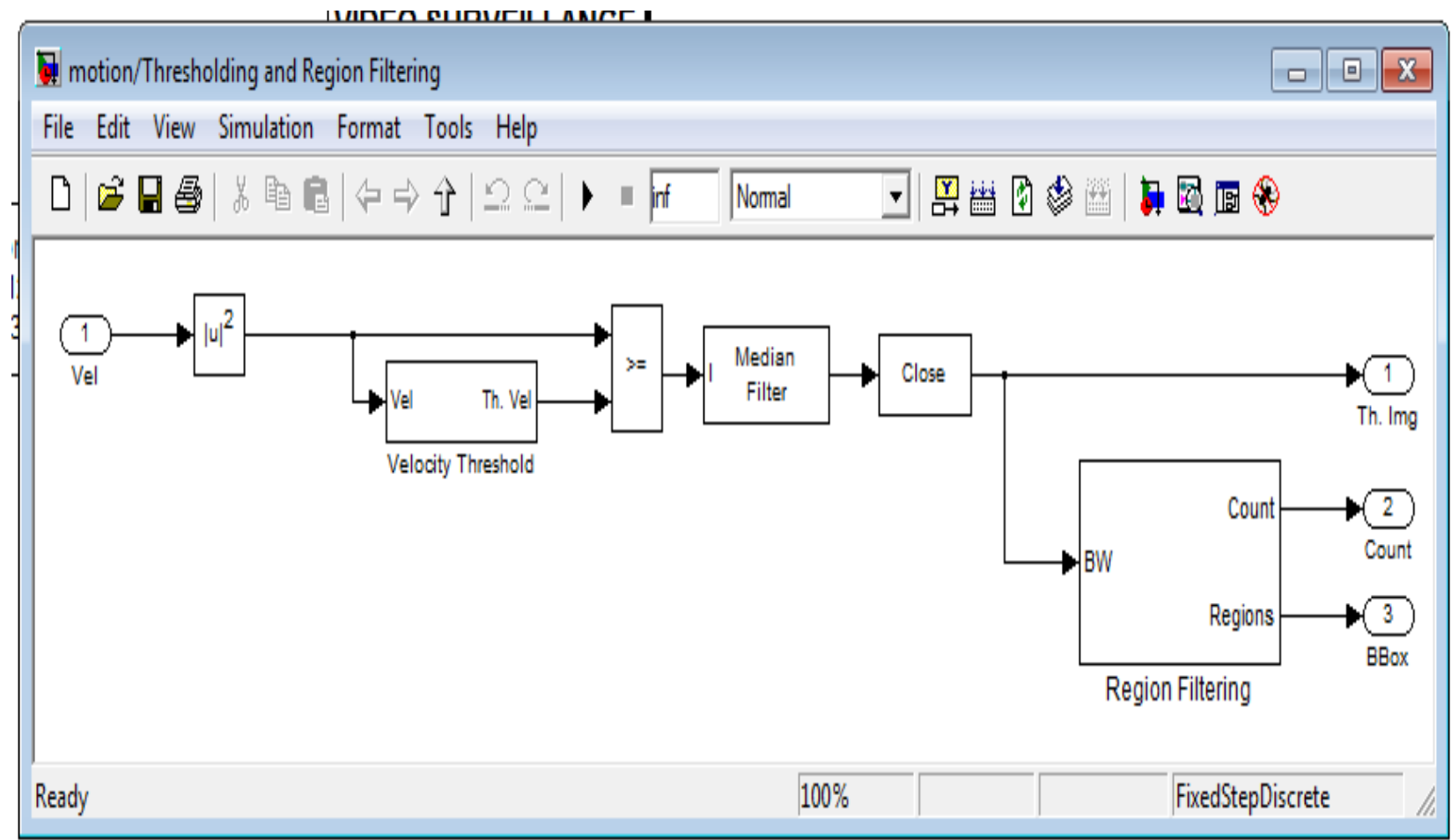

Fig.6: Simulink block of Thresholding \& Region Filtering 


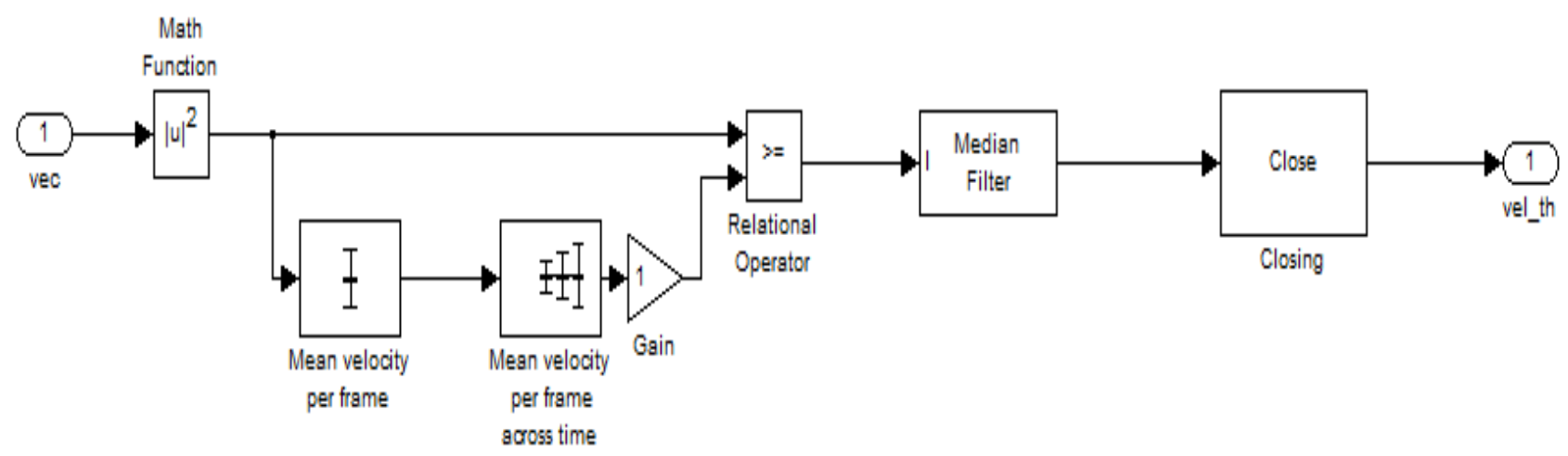

Fig.7: Subsystem that determines threshold

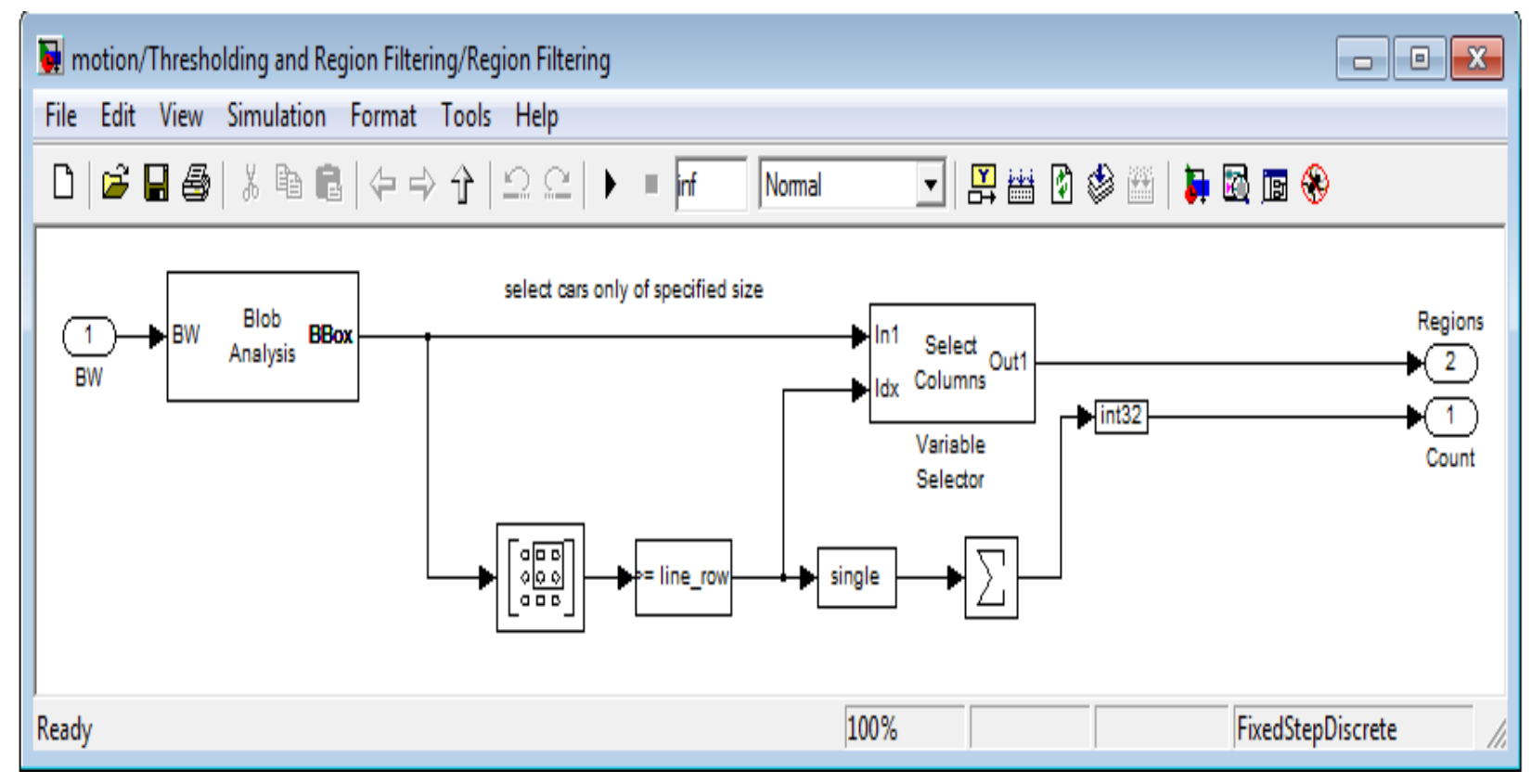

Fig 8: Subsystem that determines Region filtering

\subsection{Thresholding}

Thresholding is a fundamental method to convert a gray scale image into a binary mask, so that the objects of interest are separated from the background. In the difference image, the gray levels of pixels belonging to the foreground object should be different from the pixels belonging to the background. Thus, finding an appropriate threshold will solve the localization of the moving object problem [10]. Here we have implemented thresholding using Otsu's approach

\subsection{Segmentation based on Region filtering}

To extract feature from the object, the image has to be subdivided into constituent parts or objects. The process of sub dividing the images into its constituent parts or objects is called image segmentation. Edge detection is used as segmentation technique. [11]

\subsection{Blob Analysis}

The Blob Analysis block is use to calculate statistics for labeled regions in a binary image.[12] The block returns some quantities, such as the Centroid as values that represent spatial coordinate locations. For information about how pixel and spatial coordinate systems are defined in Video and Image Processing [13]. The Blob Analysis block processes the binary frames for use in both systems monitoring and tracking. 


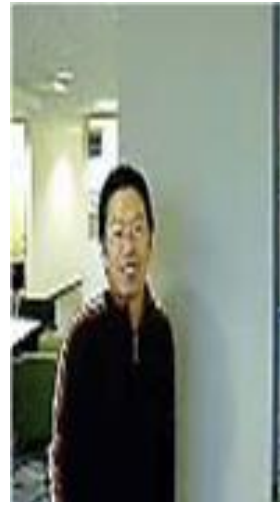

(a)

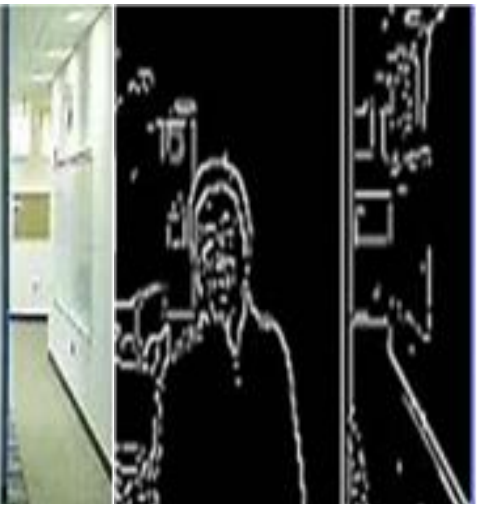

(b)
Fig 9: Segmentation based on edge detection: Canny Operator (a) Original Video (b) Moving object detection by canny method

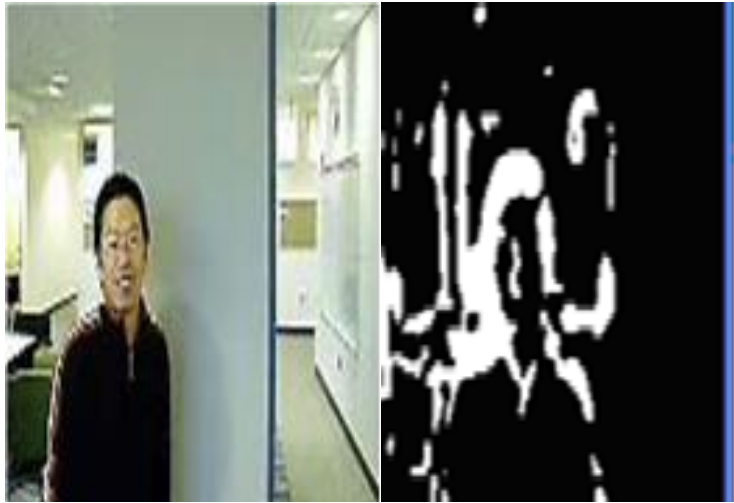

(a)

(b)

Fig 10: O/P of Thresholding: (a) Original video, (b) Foreground of the change in video is detected.

\section{FINAL SIMULINK BLOCK}

This final block of simulink of our Video surveillance system shown in Fig. 11 can track object in both abrupt change video as well as Gradual change video. Fig.12 shows the result of video surveillance system for Gradual change video and Fig.13 shows the result of video surveillance system for abrupt change video.

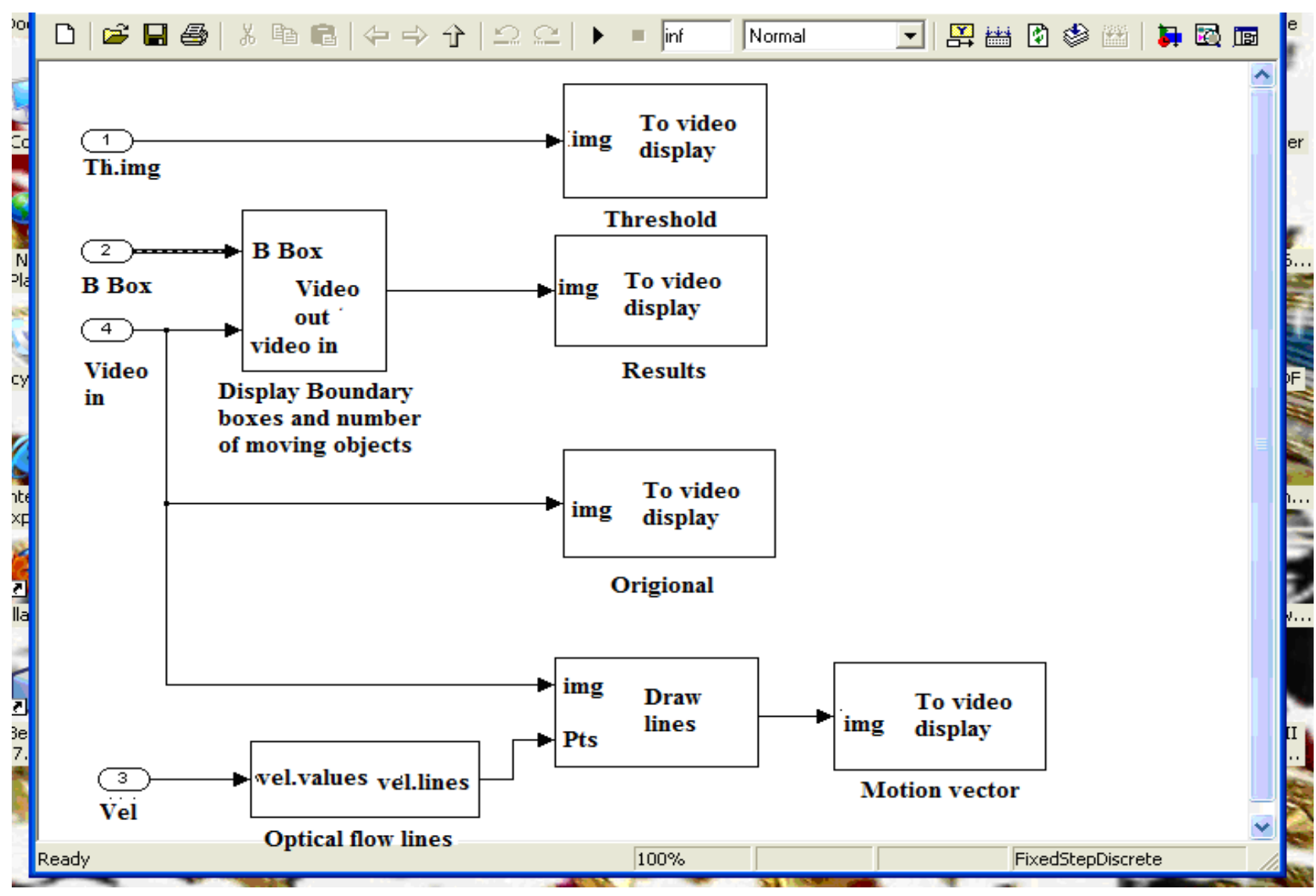

Figure 11: Object Tracking through Video surveillance system 


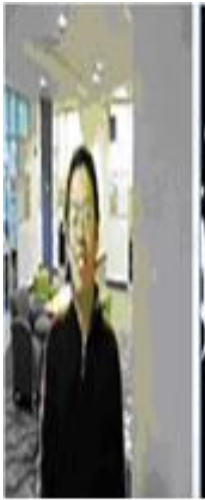

(a)

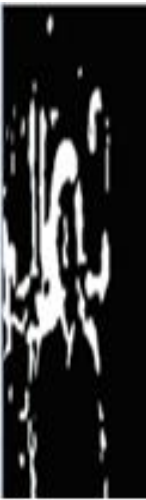

(b)

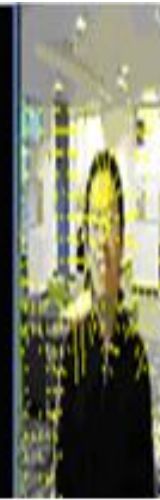

(c)

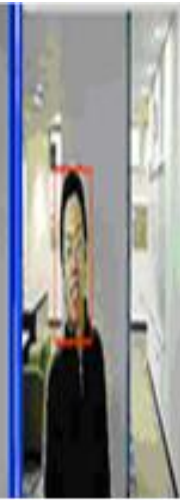

(d)
Figure: 12 : Object Tracking and Detection for video surveillance system in Gradual change video (a) shows the original video, (b) shows the threshold of moving objects,(c) shows optical flow method to set the motion vector (d) Foreground of the moving object is tracked.

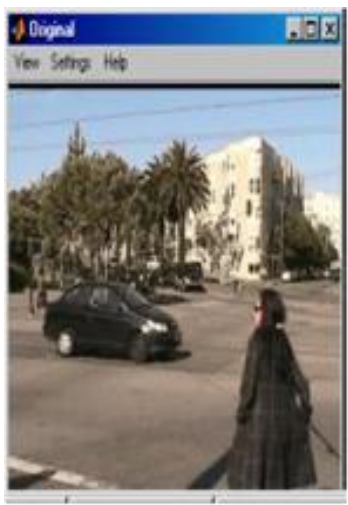

(a)

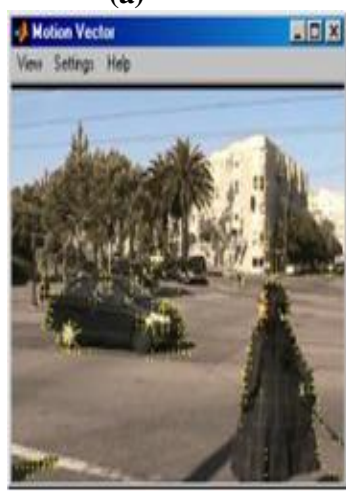

(c)

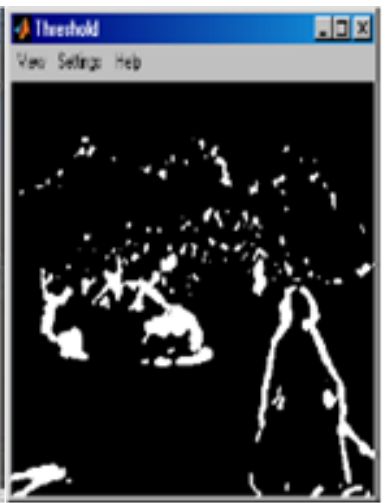

(b)

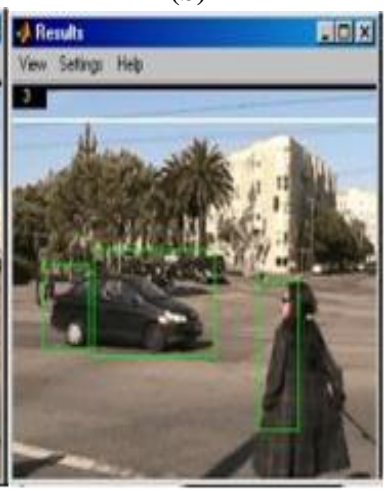

(d)

Figure: 13 : Object Tracking and Detection for video surveillance system in Abrupt change video (a) shows the original video, (b) shows the threshold of moving objects,(c)shows optical flow method to set the motion vector (d) Foreground of the moving object is tracked.

The evaluation of the proposed method is performed by comparing with other methods and the ground truth. For this reason we employ the "recall" and "precision" ratios:

Recall $=\frac{N c}{N c+N m}$
Precision $=\frac{N c}{N c+N f}$

Where $N c$ is the number of correct detections, $N f$ the number of false ones and $\mathrm{Nm}$ the number of missed ones.

So through these Recall and precision value we evaluate that proposed video surveillance system gives better performance for both Abrupt scene change video as well as Gradual scene change video. Result of scene change detection for abrupt and gradual video is shown in table 1 and table 2 respectively.

Table 1. Result of scene change detection for Abrupt video

\begin{tabular}{|c|c|c|c|c|c|}
\hline $\begin{array}{c}\text { (a)Abrupt } \\
\text { video }\end{array}$ & True & False & Missed & Recall & Precision \\
\hline $\begin{array}{c}\text { Fixed } \\
\text { Threshold }\end{array}$ & 29 & 10 & 7 & .8 & 0.74 \\
\hline $\begin{array}{l}\text { Dynamic } \\
\text { Threshold } \\
\text { without } \\
\text { exponential } \\
\text { decaying }\end{array}$ & 35 & 2 & 1 & 0.97 & 0.94 \\
\hline $\begin{array}{l}\text { Dynamic } \\
\text { Threshold } \\
\text { with } \\
\text { exponential } \\
\text { decaying }\end{array}$ & 35 & 1 & 1 & 0.97 & 0.97 \\
\hline
\end{tabular}

In Case of Abrupt scene change Condition this method reduces the false rate from 10 to 2 as we move from fixed threshold to dynamic threshold and its Recall and Precision values are 0.97 and 0.94 . According to value of Recall and precision the system performance for Abrupt scene change detection is $97 \%$.

Table 2. Result of scene change detection for Gradual video

\begin{tabular}{|c|c|c|c|c|c|}
\hline $\begin{array}{c}\text { (b) } \\
\text { Gradual } \\
\text { video }\end{array}$ & True & False & Missed & Recall & Precision \\
\hline $\begin{array}{c}\text { Fixed } \\
\text { Threshold }\end{array}$ & 43 & 12 & 17 & 0.67 & 0.78 \\
\hline $\begin{array}{c}\text { Dynamic } \\
\text { Threshold } \\
\text { without } \\
\text { exponential } \\
\text { decaying }\end{array}$ & 60 & 6 & 4 & 0.93 & 0.91 \\
\hline $\begin{array}{c}\text { Dynamic } \\
\text { Threshold } \\
\text { with } \\
\text { exponential } \\
\text { decaying }\end{array}$ & 60 & 6 & 4 & 0.93 & 0.91 \\
\hline
\end{tabular}


In Case of Gradual scene change Condition Project reduces the false rate from 12 to 6 as we move from fixed threshold to dynamic threshold and its Recall and Precision values are 0.93 and 0.91. According to value of Recall and precision the system performance for Gradual scene change detection is $93 \%$.

\section{CONCLUSIONS \& RESULTS}

The problem of tracking objects in a scene hinges on two main factors: the speed to process the video in real-time and the accuracy to distinguish between contacts moving in the scene. In this paper we have shown that through use of Optical Flow Analysis and Region Filtering it is possible to track contacts through a scene in real-time and identify some basic contact behaviors in both Abrupt change as well as Gradual change video. The Optical Flow Analysis is an iterative process to determine the best approximation to the optical flow between video frames.

The objective of tracking is to associate target objects in consecutive video frames. Videos and images from open source can be accomplished and so it can be easily implemented and its usage is wider. Implementation of this methodology using Simulink blockset is more useful for security and video surveillance. This system also provides additional services such as information about the location and identity of objects at different points in time is the basis for detecting.

\section{ACKNOWLEDGMENTS}

Our thanks to the experts who have contributed towards development of the paper. Working on this paper has been a great learning experience for me. It will be my pleasure to acknowledge, utmost co-operation \& valuable suggestion from time to time given by other staff members of Department.

\section{REFERENCES}

[1] McKenna S. and Gong S. 1999. Tracking colour objects using adaptive mixture models. Image Vision Computer vol.17, 225-231.

[2] Zhou, Q. and Aggarwal, J. K.2001. Tracking and classifying moving jects from video. Proc of 2nd IEEE Intl Workshop on Performance Evaluation of Tracking and Surveillance (PETS'2001), Kauai, Hawaii, USA .

[3] Brown, L.M. 2004. View independent vehicle/person classification. Technical report, Proceedings of the ACM 2nd international workshop on Video surveillance \& sensor networks, 114-123.

[4] Gagandeep Kaur, Sumeet Kaur. 2006. Moving Object Detection for surveillance Using Graph's Axis Change Method.ISSN:2229-6093. Vol 3 (2), 701-704.
[5] Elhabian, Shireen.Y. and Ahmed, Sumaya.H. 2009. "Moving object detection in spatial domain using background removal techniques", Recent patents on computer science, vol. 1.

[6] C.L.Huang and B.Y.Liao. 2001. A Robust Scene-Change Detection Method for Video Segmentation. IEEE Transactions on Circuits and Systems for Video Technology, vol. 11, no. 12, pp. 1281-1288.

[7] Madhur Mehta, Chandni Goyal and R.C. Jain. 2010. Real time object detection and tracking: Histogram matching and kalman filter approach. IEEE Vol 1, 4244-4260.

[8] Y.Ramadevi and B.Kalyani. 2010. " Synergy between Object Recognition and Image Segmentation", International Journal on Computer Science and Engineering, Vol. 02, No. 08, 2767-2772.

[9] P.Subashini,M.Krishnaveni. 2011. Implementation of Object Tracking System Using Region Filtering Algorithm based on Simulink Blocksets. International Journal of Engineering Science and Technology (IJEST), Vol.3 No.8. PP-6744-6750.ISSN:0975-5462.

[10] Omar Javed. 2002. "Tracking and object classification for automated surveillance". Technical report, Proceedings of the 7th European Conference on Computer Vision- Part IV, pages 343-357.

[11] Zhulin Li, Chao Xu and Yan Li. 2007. "Robust object tracking using mean shift and fast motion estimation", Intelligent Signal Processing and Communication Systems, ISPACS.

[12] Collins, R. T.; Lipton, and J. Kanade. 2000. "A system for video surveillance and monitoring", Technical Report CMU-RI-TR vo1 5, 554-572.

[13] N.Senthilkumarn and R.Rajesh. 2009.'Edge Detection Techniques for Image Segmentation- A Survey of Soft Computing Approaches", IJRTE, vol1,No2, 250-254.

[14] LeFloch $\quad$ D. 2007. Real-time people counting system using video camera. Master's thesis, Gjoevik University College, Universite de Bourgogne.

[15] Quian, Zhen. and Huang, Debao. 2011. "Moving objects detection based on Space Vector Difference", IEEE, International conference of Mechatronics and Automation, vol.646-651.

[16] Kapotas K and Skodras A. N. 2010.” Moving object detection in the H.264 compressed domain", International Conference on Imaging systems and techniques, pp. 325-328.

[17] Shafie, A. and Hafiz. 2009. "Moving object detection using optical flow", World Academy of science and technology, vol. 559-561. 